\title{
EDITORIAL
}

\section{Evaluation of Cardiac Patient Undergoing non-cardiac Surgery}

\begin{abstract}
Rationale for risk assessment
Cardiologists are often asked to evaluate cardiovascular patients who are undergoing non-cardiac surgery in daily practice. Prevalence of cardiovascular disease (CVD) increases with age. Number of non-cardiac surgical procedures in older persons will increase every year. A significant portion of these will be major intra abdominal, thoracic, vascular \& orthopaedic procedures and associated with significant perioperative cardiovascular morbidity and mortality.
\end{abstract}

\section{Pathophysiology of ischaemia during surgery}

Every surgery elicits a stress response. Fluid shifts during surgery add to the surgical stress thereby increasing myocardial oxygen demand. Surgery results in hypercoagulability. In the perioperative period due to metabolic demand chronic mismatch in the supply to demand ratio resembles stable ischaemic heart disease (IHD). Vascular inflammatory processes can lead to coronary plaque rupture presenting as acute coronary syndrome (ACS).

\section{Cardiac risk index}

Among several risk scores, Lee's revised cardiac risk index is recommended in the European Society of Cardiology (ESC) guidelines. The clinical variable in Lee's cardiac risk index include high risk surgery, IHD, congestive heart failure (CHF), history of cerebrovacular disease, insulin requiring diabetes, serum creatinine $>180 \mathrm{micromol} / \mathrm{L}$ each scoring 1 point. A score of 0 is considered very low risk with a perioperative risk of complication of $0.4 \%$ whereas a score of $>3$ is considered high risk and has $11 \%$ risk of complication.

\section{Estimating functional capacity}

Functional capacity is critical in evaluation of patient measured in metabolic equivalent (METS). Ability to perform activities of daily living can help estimate METS. A person who cannot climb 2 flight stairs has ( $<4$ METS) poor functional capacity while a person who can swim (>10 METS) has good functional capacity. If a person can achieve $>4$ METS prognosis is excellent even in the presence of stable angina or multiple risk factors. One who achieves $<4$ METS needs further risk assessment.
Exercise testing provides objective assessment of functional capacity.

\section{Patient Factors}

Cardiac risk assessment is influenced by patient factors and surgical factors. Patient factors include major predictors, intermediate predictors \& minor predictors. Major predictors include ACS, decompensated CHF, significant arrhythmias and severe valvular heart disease. Major predictors mandate intensive management and may delay surgery unless urgent.

Intermediate predictors include mild angina (CCS I or II), prior myocardial infarction (MI), compensated CHF, DM, chronic renal insufficiency which enhances risk of perioperative cardiac complications justifying further diagnostic testing and perioperative management.

\section{Diagnostic testing}

Resting ECG is recommended for patients undergoing intermediate or high risk surgery. Rest echocardiography is indicated for patients undergoing high risk surgery. Left ventricular ejection fraction (LVEF) $<35 \%$ has a sensitivity of $50 \%$ \& specificity of $91 \%$ for prediction of perioperative non fatal myocardial infarction or cardiac death. Exercise treadmill test is the preferred non-invasive stress test which has good negative predictive value. Semi-quantitative nuclear myocardial perfusion imaging (MPI) if normal indicates an excellent surgical prognosis. Dobutamine stress echocardiography (DSE) has good negative predictive value (similar to MPI). Coronary angiogram (CAG) is considered for facing high stress surgery who have ACS or decompensated ischaemic CHF.

Intraoperative pulmonary artery catheter or transoesophageal echocardiography (TOE) may be helpful in ischaemic cardiomyopathy.

\section{Surgical factors}

High risk surgery (cardiac risk $>5 \%$ ) include emergency major operations especially in elderly, aortic \& other major vascular surgery, peripheral vascular surgery, anticipated prolonged surgical procedures associated with large fluid shift and/or blood loss. Intermediate risk surgery (cardiac risk 1-5\%) includes carotid endarterectomy, head \& neck 
surgery, intraperitoneal \& intrathoracic surgery, orthopaedic \& prostate surgery. Laparoscopic procedures carry a similar cardiac risk to open procedures.

Validated pharmacologic therapies

Beta-blockers (BB) with a target heart rate of 60-70/minute reduce perioperative myocardial ischaemia, $\mathrm{MI}$ and cardiac mortality seen in large meta-analyses. They should not be withdrawn preoperatively. Intermediate and high risk patients should be stabilised with on BBs prior to surgery. Observation data suggests perioperative use of statin offers protection against cardiac complications. In patients with risk factors statins should be initiated preoperatively. Some data suggests use of ACE inhibitors reduce postoperative cardiovascular events. It is recommended to commence and continue ACE inhibitors in patients with reduced LV function but transient discontinuation of ACE inhibitors prescribed for hypertension should be considered before surgery.

\section{Consideration of revascularization}

Stress testing may be useful in highest risk patients undergoing highest risk surgical procedure if the outcome will affect management. Intermediate risk patients need optimal medical management. Prophylactic revascularization is not recommended in patient s with stable angina. Post- surgical revascularization recovery before non-cardiac surgery is a matter of expert opinion which is not evidence or guideline based. Use of drug eluting stent (DES)/bare metal stent needs to be considered in view of dual antiplatelet therapy (DAPT). Use of perioperative intraaortic balloon pump should be considered in high risk cases. In patients with previous revascularization with DES undergoing surgery, single antiplatelet should continue following mandated period of DAPT.

\section{Dr. Manzoor Mahmood and Dr. Harisul Hoque \\ Associate Professor \\ Department of Cardiology \\ Bangabandhu Sheikh Mujib Medical University}

\section{References:}

2014 ACC/AHA Guideline on Perioperative Cardiovascular Evaluation and Management of Patients Undergoing Noncardiac Surgery. : A Report of the American College of Cardiology/American Heart Association Task Force on Practice Guidelines

2014 ESC/ESA Guidelines on noncardiac surgery: cardiovascular assessment and management. European Heart Journal doi: 10.1093/eurheartj/ehu282. 